\title{
Analysis of Load Balancing and Fault Tolerant Routing Protocol for Wireless Sensor Network
}

\author{
${ }^{* 1}$ Roshani Talmale, ${ }^{2} \mathrm{M}$. Nirupama Bhat, ${ }^{3}$ Nita Thakare \\ ${ }^{1,2}$ Vignan's University, Guntur, A.P \\ ${ }^{3}$ Priyadarshani College of Engineering, Nagpur \\ Email: roshanikambe@rediffmail.com,nirupamakonda@gmail.com,nitathakare14@gmail.com
}

Received: 09 ${ }^{\text {th }}$ July 2018, Accepted: $14^{\text {th }}$ August 2018, Published: 31 ${ }^{\text {st }}$ August 2018

\begin{abstract}
Fast development from last few years in wireless senor network has create great impact on researcher and motivated by various real time issues. There are huge numbers of sensors deploy in network which sense the neighbouring information and processed the gathered data to the base station, processing speed, storage and communication bandwidth. Because of these constraints fast transmission, fault tolerant, load balancing mechanism and energy efficient routing are critical requirements in WSNs to increase the network lifetime. By considering this limitation it is necessary to design novel energy aware and fault tolerant routing protocol for heterogeneous sensor network. In this survey paper various existing techniques are studies and list out the research challenges.
\end{abstract}

Keywords: Routing, Energy Efficient, Fault Tolerant

\section{Introduction}

Efficient Energy utilization and load balancing in densely deploy heterogeneous sensor network is a big challenge [1]. Wireless Sensor Network (WSN) is consisting small sensors which is having very low battery power. These sensor nodes sense the information, performed data fusion at sink node and sent combined information to a base station (BS). Sensor is having limited power capacity and the battery replacement is not easily possible. Hence for long life functioning of wireless sensor network it is necessary to design energy efficient routing protocol. To achieve this goal, we required to design novel techniques which will helps to minimize total energy consumption and to balance the load of gateway/relay node or cluster head $(\mathrm{CH})$. One important contribution to reduce energy consumption is to design a sensor that will operate on lower technologies and will consume less energy. Designing low power chip is the good technique but if sensor components are unable to operate properly then the complete sensor network will break. Wireless sensor network must work at least for a targeted area for longer period of time. Because of hardware failure sensor node might be damage or run away from transmission range, since the wireless communication between two nodes can be permanently interrupted. It is necessary to tolerant such faults otherwise it will affect complete network. To support long life of sensor, energy efficient operation is a key technique. Enhanced the network life time of a WSN becomes very challenging and this issue need to be consider. In order to achieve the energy efficient operation in sensor network the main focus has to give on proper distribution of load to cluster head and balance the energy consumption of individual nodes to the highest possible extent. The existing techniques used to achieve these objectives are based on parameter such as load balancing, data aggregation, change the transition state of sensor (active and sleep mode) and efficient cluster formation with cluster head rotation as per balance energy [1] [2].

Various type of clustering technique is used for energy efficient routings. Sensor node are grouped in to cluster, each sensor node belongs to only one cluster. Each cluster has a Cluster Head $(\mathrm{CH})$ which will collect the information from its sensor node, aggregate them and forwarded the sensing information to the base station (BS). Being a cluster head means taking over additional task. Hence the battery of cluster head will exhausted very soon. Therefore many researchers [3] have proposed that $\mathrm{CH}$ responsibility should be rotate. Clusters formation algorithm cannot do only once but it must execute after periodic time so that the load of cluster head will share and helps to save the energy. For selecting the cycle of cluster formation and cluster head we have to consider the average data transmission and reception speed of node, battery draining rate and residual energy etc. Many clustering techniques have been implemented so far. Authors [2] proposed the method the gateway or relay called as a cluster head. The cluster head provided a more energy and higher communication range than other sensor. However the gateways are also power constraints.

Another issue with the WSN is data aggregation. The huge number of sensor nodes gathered the surrounding information. Each sensor node required sufficient energy to transmit and receive the data generated by surrounding sensors. Mostly in densely distributed and heterogeneous network, the life of sensors is very short because of heavy load. In order to resolve these issues, we need energy-efficient method to aggregate huge data and load balancing mechanism to increase the network life time. 
There are few documents which worked to tackle the challenges of distributed sensor network related to efficient load balancing and fault tolerant with less energy consumption.

\section{Literature Survey}

The various clustering techniques and routing protocols proposed in many literatures. The first protocols to implement clustering techniques which select the Cluster Head on periodic rotation basis is LEACH [4] protocol. In this method it assumes that all the nodes can communicate with the $\mathrm{CH}$ (BS) and share information to each other. It minimizes transmission range within cluster and save energy. The parameter consider for selection of cluster head is signal strength and distance of particular sensor node from the base station.

Many authors have proposed the selection of cluster head $(\mathrm{CH})$ is based on remaining residual energy with that particular sensor node. Second parameter is amount of traffic can be handling, number of neighboring node and density of network. Cluster Head rotation is performed by using IEEE 802.15.4 LR WPAN protocol [3]. Clustering is performed only at the initial stage and it will not change for particular time period. Dead time is calculated and overhead is minimizing. In case of $\mathrm{CH}$ fails, the other sensor node will notice that there is no cluster head to synchronize the data. After a predefine time slot, sensor report to BS and assign orphaned node to the cluster. Assign new orphaned node will increase the traffic. In such case BS will monitor the traffic. The issue with this technique is sensor node has to wait for predefined time and then it is informed to $\mathrm{BS}$, since it will create a delay in transmission of sensed data.

In [5] authors proposed distributed and hybrid clustering algorithm which is energy efficient. It calculates the energy of each node and selects the cluster head which is having more energy. Load balancing is performed by rotating cluster head. But by repeated selections of cluster head more energy can be consume.

The author [6] presented a method based on TreeletBased Clustered. Tree structure is constructed and cluster head is always a root of the tree. Shortest distance node from cluster head becomes the child of a tree. Cluster head is having more power of transmission and energy than the other node.

Energy balanced routing protocol (GSTEB) proposed by author in [7]. In proposed system BS assign a root node. Path information and ID is broadcast by base station to create a new path from sensor node. In proposed techniques topology remain same until the root dies. Once the root dies then new tree is constructed.

The authors [8] presented dual path geographic routing aim to design geographic routing to transfer the data by passing the routing hole. In this technique the forwarder list is prepared. It prepared two different forward lists to transfer the data to destination in case of node failure. This is based on the assumption that the nodes are aware about the actual and alternate virtual location which can be calculated using Global Positioning Service. While transferring the data if there is no node in the relay region of the current forwarder list then introduce a random shift to continue the data delivery. The proposed algorithm is focus on energy efficiency but no energy balancing is addressed in routing phase. To enhance the network lifetime node balancing plays very important role.

In [9][10] authors suggested PSO encoding scheme. It selects an energy efficient path by considering transmission distance from sensor node to base station and amount of data available. The selected path is energy efficient but not energy balanced because path is remains static throughout the network lifetime. Some cluster head face the problem of heavy routing load and deplete their energy very soon. The authors apply the clustering techniques to form the cluster by considering routing over head of the $\mathrm{CH}$ s for the selected path and assign least number of sensor nodes to the heavily loaded $\mathrm{CH}$ for balancing the load. This may slightly reduce the energy consumption of cluster head. The proposed method setup the route and forms clusters. The Base Station first construct a hop tree where each gateway is assign a hop value to set up the route. To identify the next hope from each $\mathrm{CH}$ the cluster is formed by using the final route setup. In this paper fault tolerant is detected by gateway if it does not receive any information. After the detection of the gateway failure, the gateway broadcast the HELP message to all sensors. All other node is sending the reply message with hope count and residue energy to the $\mathrm{CH}$. The algorithm selects new cluster head with value $n-1$.This approach is focus only on gateway failure but in case if other than $\mathrm{CH}$ node will fail then the delay occurs in transmission. The author proposed distributed data aggregation Technique to combine the collected data from sensor nodes [10]. This algorithm combines the incoming messages at sink node and transfer the combined one to base station but at the same time it required extra memory and high computation power. This method is not suitable for densely deploy sensors node. Sensor nodes gathered huge amount of redundant data. For data aggregation similar type of packets from multiple sources can be combined so that the number of transmissions is reduced. The author proposed data aggregation technique that has been implemented to achieve energy efficiency operation and optimized data transfer using routing protocols. Data compression and signal processing techniques can also be used for data summarization. Data aggregation is important parameter which will reflect on less energy consumption processing by avoiding duplicate transmission .It is an efficient method to balance the load of sensors and required less energy by eliminating repeated transmissions. 
In wireless sensor network data fusion is an efficient technique to avoid repeated transmission. By avoiding repeated transmission of data energy consumption can be minimize and the utilization of wireless channel will be improved, but on other hand energy is also consumed while performing data aggregations. So designing efficient and reasonable data aggregation algorithm is one of the most important research fields to enhance the energy aware routing in WSNs' performance. A novel data aggregation algorithm Dynamic Queue Data Aggregation is proposed by author in [11]. This technique is based on the hierarchical routing algorithm. A dynamic queue is defined in every sensor node to store history messages transmitted by this node, when new message is received then it will examine whether the message is duplicate or not.

In [12] authors proposed Location Aware Data Clustering (LABDC) algorithm. The designed model minimizes the number of transmissions and its cost. One of the issues in this algorithm is that it is efficient in highly correlated sensor. Cheng Zhao et. al. proposed data aggregation on Treelet Based Clustered for Wireless Sensor Networks, which implement data fusion and cluster formation techniques using tree based method. Load balancing issue is not considering in both cases.

\begin{tabular}{|c|c|c|c|}
\hline Authors & $\begin{array}{c}\text { Techniques } \\
\text { used }\end{array}$ & $\begin{array}{c}\text { Advantages } \\
\text { in WSN }\end{array}$ & Limitations \\
\hline $\begin{array}{l}\text { Haiying } \\
\text { Shen } \\
\text { et.al.[2] }\end{array}$ & $\begin{array}{c}\text { Consider } \\
\text { gateway/relay } \\
\text { as a cluster } \\
\text { head }\end{array}$ & $\begin{array}{c}\text { Alternate } \\
\text { routing path } \\
\text { is available in } \\
\text { case of node } \\
\text { failure } \\
\end{array}$ & \begin{tabular}{|} 
Cluster formation is \\
based on shortest \\
distance. Energy \\
balance issue is not \\
considered \\
\end{tabular} \\
\hline $\begin{array}{l}\text { Hamidre } \\
\text { za } \\
\text { Tavakoli } \\
\text { et. al. [3] }\end{array}$ & $\begin{array}{l}\text { Cluster head } \\
\text { selection is on } \\
\text { rotation basis } \\
\text { by } \\
\text { considering } \\
\text { energy factor }\end{array}$ & $\begin{array}{c}\text { Load } \\
\text { Balancing } \\
\text { issue is } \\
\text { implemented }\end{array}$ & $\begin{array}{l}\text { In case of node } \\
\text { failure alternate path } \\
\text { is not available in } \\
\text { advanced. It find } \\
\text { alternate path once } \\
\text { fault is detected }\end{array}$ \\
\hline $\begin{array}{l}\text { O.Youni } \\
\text { s and S. } \\
\text { Fahmy } \\
\text { [5] }\end{array}$ & $\begin{array}{c}\text { Hybrid } \\
\text { energy } \\
\text { efficient \& } \\
\text { Distributed } \\
\text { techniques is } \\
\text { used }\end{array}$ & $\begin{array}{c}\text { Cluster head } \\
\text { assign more } \\
\text { energy and } \\
\text { transmission } \\
\text { power }\end{array}$ & $\begin{array}{c}\text { Because of heavy } \\
\text { load at cluster head } \\
\text { node exhausted soon }\end{array}$ \\
\hline $\begin{array}{l}\text { Cheng } \\
\text { Zhao } \\
\text { et.al.[6] }\end{array}$ & $\begin{array}{c}\text { Tree based } \\
\text { clustering and } \\
\text { data } \\
\text { aggregation is } \\
\text { used }\end{array}$ & $\begin{array}{c}\text { Avoid } \\
\text { repeated } \\
\text { transmission } \\
\text { and save } \\
\text { energy }\end{array}$ & $\begin{array}{l}\text { Cluster head is root } \\
\text { of the tree and } \\
\text { distance is consider } \\
\text { as a parameter to } \\
\text { construct the not } \\
\text { energy of node }\end{array}$ \\
\hline $\begin{array}{c}\text { Zhao } \\
\text { Han et. } \\
\text { al. [7] }\end{array}$ & $\begin{array}{c}\text { Energy } \\
\text { balance } \\
\text { routing tree is } \\
\text { used }\end{array}$ & $\begin{array}{c}\text { Load } \\
\text { balancing } \\
\text { techniques is } \\
\text { used }\end{array}$ & $\begin{array}{c}\text { Strategy remains } \\
\text { same until root node } \\
\text { die. }\end{array}$ \\
\hline $\begin{array}{c}\text { Md } \\
\text { Azharud } \\
\text { din et.al. } \\
{[9][10]}\end{array}$ & $\begin{array}{l}\text { Distributed } \\
\text { energy } \\
\text { balanced } \\
\text { algorithm is } \\
\text { used }\end{array}$ & $\begin{array}{c}\text { Selects } \\
\text { energy } \\
\text { efficient path } \\
\text { by } \\
\text { considering } \\
\text { shortest } \\
\text { distance }\end{array}$ & $\begin{array}{c}\text { Selected path is } \\
\text { energy efficient but } \\
\text { not energy balanced. } \\
\text { Selected path is } \\
\text { remain static }\end{array}$ \\
\hline
\end{tabular}

Table1: Comparisons of Energy Efficient Routing Techniques
Summary: Table 1 shows the comparative study of energy efficient routing techniques for WSNs. Many authors has been taken extensive efforts to design a protocol which is suitable for energy efficient load balancing and fault tolerant routing in WSNs, still there are some scope to enhance the performance and generate effective solutions to fault tolerant and energy aware routing which will helps to improve the quality of service for sensor network.

\section{Discussion}

In real time applications, it is highly important to deliver a data to the destination within a certain period of time span from the time it is sensed or collected; otherwise unable to take realistic decisions. Therefore fast transmission of data from source to destination is another condition for timeconstrained applications. However, in many papers, energy conservation is considered relatively more important than the quality of data sent. As the energy gets discharge, the network may reduce the quality of the results. In order to focus on these issues we required energy-aware routing protocols to fulfill the target.

Although the performance of the existing protocols [12] [13] is efficient in terms of energy efficiency, still more focus would be needed to address the issues like fast data transmission in real-time applications, Efficient Energy-aware routing, fault tolerant routing. Another issue for routing protocols is the consideration of node mobility. In real time application there might be situations where the base stations and possibly the sensors are in mobile state. In such a scenario, the rapid update of the position of the cluster head and the sensor nodes is required. Design an innovative and energy efficient routing techniques is needed in order to handle the overload of mobility and topology changes in such heterogeneous and dynamic sensor environment. This inspires to outline a novel and efficient algorithm for better use of energy, fault tolerant and load balancing in WSN. In this paper various existing energy saving and fault tolerant techniques for WSN are studied and some research challenges related to the adoption of existing and traditional techniques are listed out.

\section{Conclusion}

We have presented a comprehensive analysis of existing techniques based on energy aware load balancing for WSNs. All analysed techniques are focus on performance parameter such as network lifetime, efficient data routing, quality of service with data accuracy and less energy consumption for homogeneous as well as heterogeneous network. It is observed that by considering the limitation of WSN it is very challenging to upgrade the quality of service of WSNs with respect to less energy consumption. This inspires to propose a novel energy efficient routing technique which will give more focus on dynamic energy management in case of fault tolerant in heterogeneous networks. 


\section{References}

1. Jamal N. Al-Karaki Ahmed E. Kama. Routing Techniques in Wireless Sensor Networks: A Survey. 2008.

2.Haiying Shen, Ze Li, A Kautz-Based Wireless Sensor and Actuator Network for Real-Time, FaultTolerant and Energy- Efficient Transmission, IEEE Transaction, 2016

3. Hamidreza Tavakoli, Jelena Mi, Vojislav B., Energy - Efficient Cluster-Head Rotation in Beacon-Enabled IEEE 802.15.4 Networks , IEEE Transaction ,2015

4. W. Heinzelman, A. Chandrakasan and H. Balakrishnan, Energy efficient communication protocol for wireless micro sensor network, IEEE Transaction, Vol 1no.4, pp 660-670,Oct. 2002.

5.O.Younis and S. Fahmy, HEED, A hybrid energy efficient distributed clustering approach for sensor network, IEEE Transaction, Mobile computing, Vol 3,no 4,pp 660-669,2004

6. Cheng Zhao, Student Member, IEEE, Wuxiong Zhang, Member, IEEE, Yang Yang, Treelet-Based Clustered Compressive Data Aggregation for Wireless Sensor Networks, , IEEE Transactions on Vehicular Technology, pp 1-10.

7. Zhao Han, Jie Wu, Member, Ieee, Jie Zhang, Liefeng Liu, And Kaiyun Tian, ,General Self Organized Tree Based Energy Balance Routing Protocol (GSTEB) For WSN, IEEE Transactions on Nuclear Science,Vol.61,No.2,April2014

8. Haojun Huang, Hao Yin, Geyong Min,Energyaware Dual-path Geographic Routing to Bypass Routing Holes in Wireless Sensor Networks, IEEE Transaction ,2017.

9. Md Azharuddin, Prasanta K. Jana, PSO based approach for energy efficient and energy balanced routing and clustering in WSN, Springer 2016,

10. Md Azharuddin, Prasanta K. Jana, Distributed algorithm for energy efficient and fault tolerant routing in wireless sensor network, Springer 2014.,10.1007/s11276-014-0782-2

11. Tao Du, Shouning Qu, Qingbei Guo, A high efficient real time data aggregation algorithm for WSNs ,IEEE Conference 2014, pp 594-598.

12.Sarvesh Kumar Singh, Rajeev Paulus et. al., Analysis of Energy model and QOS in wireless Sensor Networks, International Conference for Convergence of Technology, 2014 IEEE.

13.Yonghui Shim, Younghan Kim ,Data Aggregation with Multiple Sinks in Information Centric Wireless Sensor Network, ICOIN IEEE 2014. 